\title{
Unusual Presentation of a Gastrointestinal Stromal Tumor in a Small Intestine Diverticulum
}

\author{
Mackenna Senti ${ }^{a}$ Tania A. Torres ${ }^{b}$ Jairo Espinosa ${ }^{b} \quad$ Saad Shebrain ${ }^{b}$ \\ aWestern Michigan University Homer Stryker M.D. School of Medicine, Kalamazoo, MI, \\ USA; bepartment of Surgery, Western Michigan University Homer Stryker M.D. School of \\ Medicine, Kalamazoo, MI, USA
}

\section{Keywords}

Gastrointestinal stromal tumor · Perforation · Diverticulum

\begin{abstract}
A 50-year-old female with no significant medical history initially presented to an urgent care center with symptoms of acute onset abdominal pain, nausea, and emesis. Chest and abdominal $\mathrm{X}$-ray revealed free air under the diaphragm, prompting immediate transfer to the emergency department. Continued abdominal tenderness and pain were concerning for perforated viscus. The patient was transferred to the operating room, and diagnostic laparoscopy was performed. Inflammation and contamination were discovered in the right side of the abdomen and pelvis secondary to a small bowel (SB) perforation. Segmental SB resection revealed a perforated diverticulum. Pathological examination confirmed a diagnosis of gastrointestinal stromal tumor (GIST) at the perforated segment. On postoperative day 5 , the patient was discharged home, and at 30-month follow-up, the patient continued to do well. Although rare, SB diverticula are commonly false (i.e., pseudodiverticula). The concomitant presence of a GIST in a true SB diverticulum presenting with perforation has not yet been reported.
\end{abstract}

\section{Introduction}

GISTs, a common mesenchymal cancer of the GI tract, develop from precursors of the cells of Cajal, a population of cells in the gastrointestinal tract with pacemaker activity [1]. GISTs may occur at any age but affect mostly middle-aged and elderly patients. A median age of diagnosis of 65 years has been reported [2]. Additionally, micro-GISTs $(<1 \mathrm{~cm})$ are common in the older population but are considered clinically insignificant with little to 

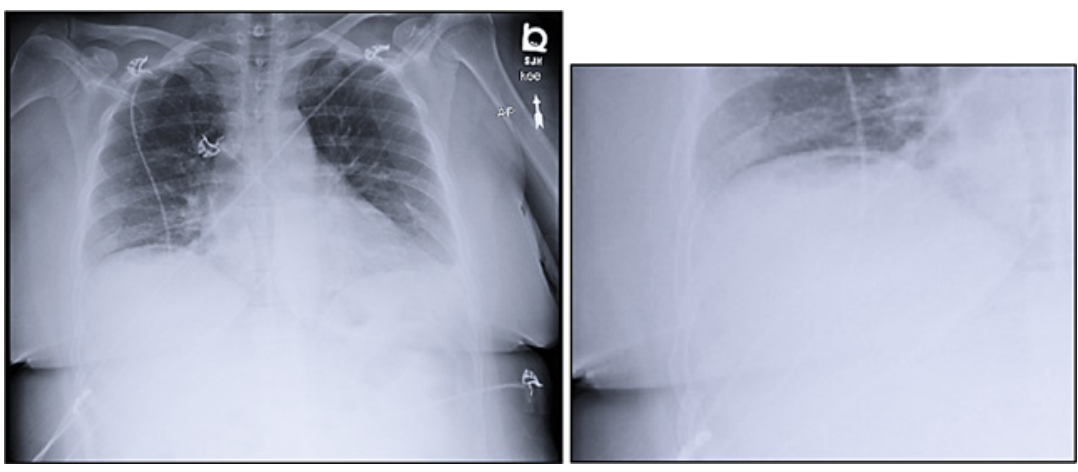

Fig. 1. X-ray image of the patient before segmental surgical resection of the small intestine demonstrating air under the diaphragm.

no mitotic activity [3]. GISTs are found within the stomach in $60-70 \%$ of affected patients and, however, may be located in the small intestine in $25-35 \%$ of affected patients $[4,5]$. Less than $5 \%$ of GISTs arise outside of the GI tract. Occurrence is equal between genders, and tumor size averages around $6 \mathrm{~cm}$ [2]. GISTs often protrude into the GI lumen or abdominal cavity and, due to their vascularity, commonly present with bleeding, resulting in anemia, tachycardia, fainting, abdominal pain, or melena [2]. About $97 \%$ of GISTs are sporadic tumors, and about $20 \%$ of affected patients present with overt metastasis at diagnosis [6, 7]. GISTs have been reported in unusual abdominal locations including the Meckel's diverticulum $[8,9]$. GI perforation at the site of the GIST is rare but has been reported $[9,10]$. We report a rare case presentation of a GIST as a perforated diverticular structure in the terminal ileum in an adult female patient with acute onset abdominal pain. According to our literature review, this is the first description of a GIST presenting as a perforated true diverticular structure within the ileum.

\section{Case Presentation}

A 50-year-old female with no significant past medical history initially presented to an urgent care center for 1-day history of acute abdominal pain associated with nausea and emesis. Medical evaluation included an upright chest X-ray and abdominal X-ray which demonstrated free air under the diaphragm (Fig. 1). The patient was immediately transferred to the emergency department.

In the emergency department, the patient's vital signs were notable for tachycardia at $110 \mathrm{bpm}$ and hypotension at a blood pressure of $105 \mathrm{~mm} \mathrm{Hg}$ systolic. Other vitals included 18 respirations per min, $98 \%$ oxygen saturation, and a temperature of $37^{\circ} \mathrm{C}$. She demonstrated signs of dehydration with dry mucosa and cool, clammy, and slightly diaphoretic skin. She had clear lungs to auscultation. Abdominal examination showed mild distension but with absence of surgical scars or other lesions. On palpation, there was diffuse tenderness more pronounced in the right side of the abdomen, right upper quadrant, and epigastric region consistent with peritonitis concerning for perforated viscus. Fluid resuscitation and parenteral antibiotics were administered, and the patient was taken emergently to the operating room. A diagnostic laparoscopy was performed and demonstrated significant inflammation and contamination with enteric contents in the right side of the abdomen and pelvis. Due to the severity of inflammation and inadequacy of visualization, a laparotomy was performed. We found a perforated small intestine diverticulum located on the mesenteric side about $100 \mathrm{~cm}$ proximal to the 


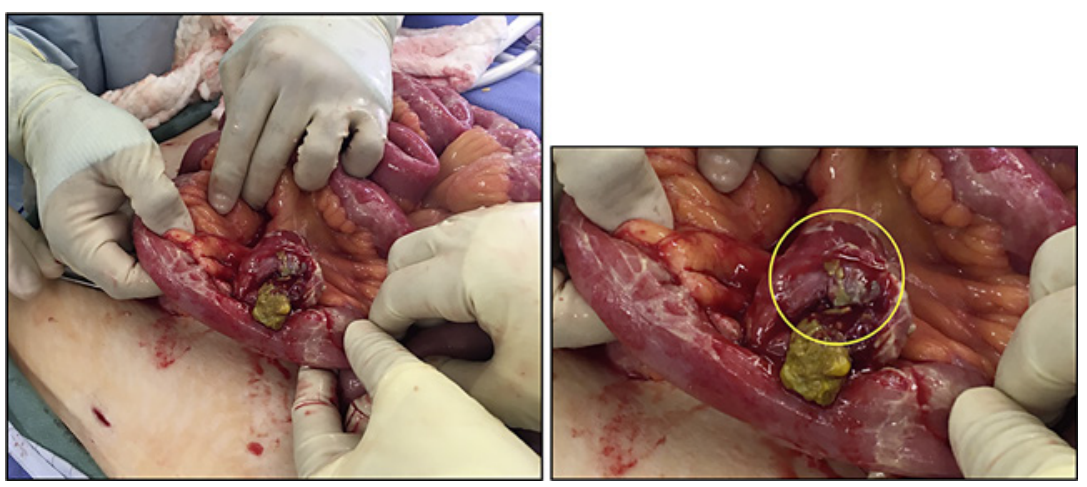

Fig. 2. Perforated small intestine diverticulum with GIST indicated.

cecum. Once the contamination was controlled, an oncologic segmental small intestine resection with adjacent mesentery with side-to-side functional end-to-end anastomosis was performed. Figure 2 displays the perforated small intestine diverticulum at the time of surgery.

Final gross pathological examination displayed a $7.5-\mathrm{cm}$ segment of small bowel with a $5.5 \times$ $3.0 \mathrm{~cm}$ outpouching consistent with a diverticulum near its midpoint. The diverticulum shows a $1.0 \times 0.7 \mathrm{~cm}$ perforation with surrounding hemorrhage, and at the tip of the diverticulum, a $2.7 \times$ $2.5 \mathrm{~cm}$ firm mass-like area was noted and is $0.8 \mathrm{~cm}$ from the perforation and $1.5 \mathrm{~cm}$ from the mesenteric margin. Microscopic examination showed a moderately cellular, predominantly spindle cell neoplasm with moderate nuclear pleomorphism. Approximately 3 mitotic figures per 50 high-power fields were noted. No tumoral necrosis was appreciated. Immunohistochemical (IHC) staining for the CD117 antigen (c-kit proto-oncogene) and DOG-1 was performed and was positive, confirming the GIST diagnosis. Stains for S-100, actin, and desmin were negative. Hematoxylin and eosin (H\&E) staining displayed characteristic spindle cells and epithelioid nature. Figure 3 displays both the H\&E and IHC staining utilized for GIST diagnosis confirmation.

The patient recovered well, and the postoperative course was uneventful. She was discharged home on postoperative day 5. At 4-month follow-up, the patient continued to do well. An upper GI endoscopy was performed and revealed no evidence of gastric or duodenal abnormalities suggestive of GIST. She continues to do well 2.5 years after surgery.

Based on tumor size and mitotic index, tumor risk categories were evaluated according to the National Comprehensive Cancer Network (NCCN) Clinical Practice Guidelines [11]. The patient was found to be low risk, and therefore postoperative chemotherapy was not indicated. Both perforated GI diverticulum and GIST are rare pathologies and when combined warrant careful consideration for optimal patient care.

\section{Discussion}

GISTs are rare tumors of the GI tract. They originate from malignant precursors of interstitial cells of Cajal and are the most common mesenchymal GI neoplasm [1]. About 20\% of soft-tissue sarcomas classify as a GIST and affect about 10 out of every 1 million people, affecting males and females equally [12]. GISTs $<1 \mathrm{~cm}$, known as micro-GISTs, are more common in the elderly population and are usually clinically insignificant [3]. Average age of diagnosis is 65 years with $>99.5 \%$ of affected patients being older than 20 years [13]. About $60-70 \%$ of GISTs present in the stomach of affected patients, but anywhere from 25 to $35 \%$ of patients present with small intestinal GIST $[4,5]$. GISTs rarely occur outside of the GI tract [2]. Nearly all GISTs are spontaneous neoplasms.

\section{Karger's}




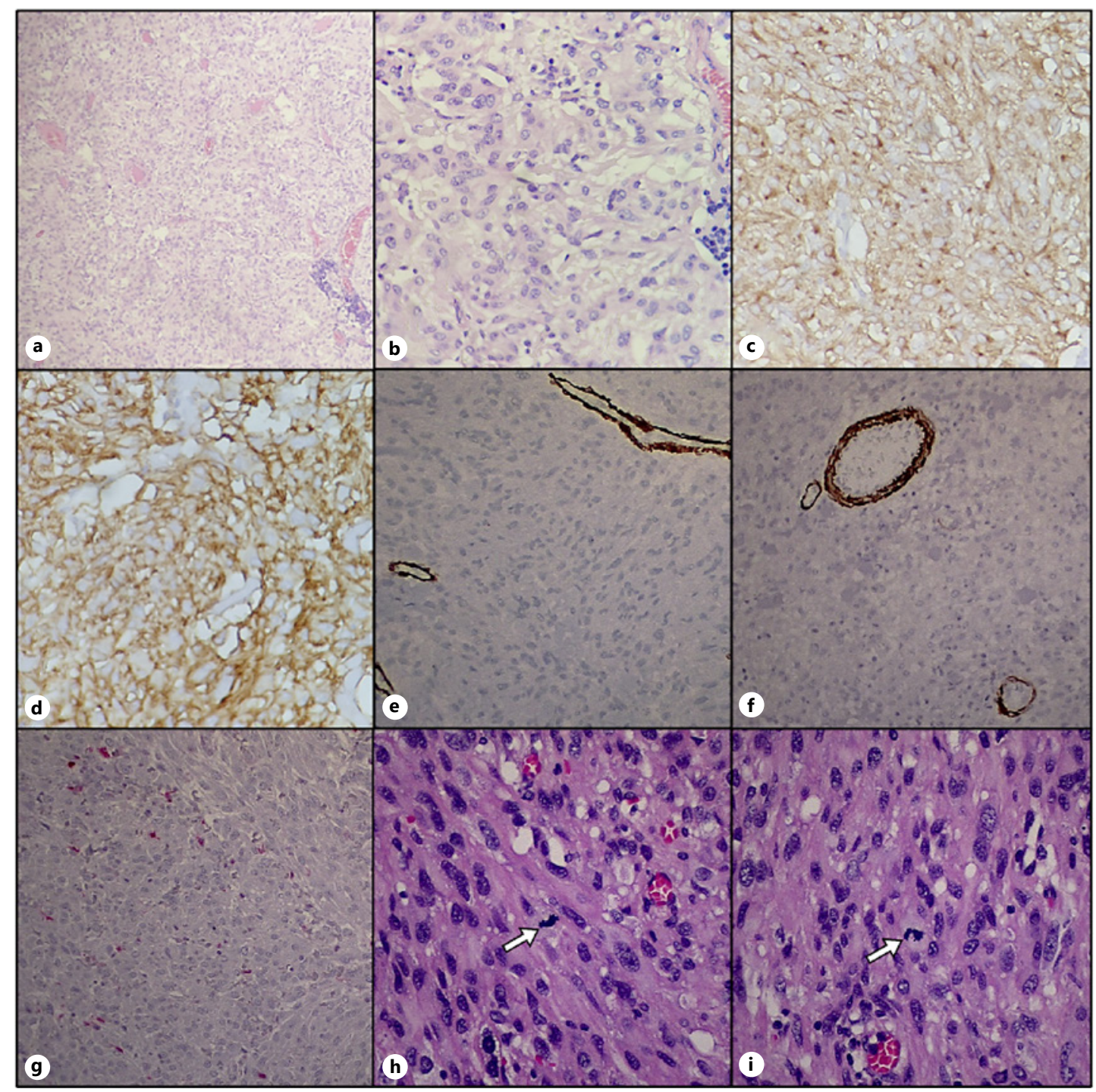

Fig. 3. $H \& E$ stain at $\times 100$ magnification (a) and H\&E stain at $\times 400$ magnification (b) displaying characteristic spindle cells and epithelioid nature. c IHC staining for CD117 (c-KIT proto-oncogene) at $\times 400$ magnification. d IHC staining for DOG-1 (chloride channel protein anoctamin 1) at $\times 400$ magnification. Negative IHC staining for actin (e), desmin (f), and S-100 (g) at $\times 400$ magnification. $\mathbf{h}$, i Mitotic figures indicated. Fifty $\times 40$ magnification fields were used to determine the number of mitotic figures. IHC, immunohistochemical.

Small bowel diverticula are rare and are commonly grouped as either true or false. False diverticula, the more common of the 2 types, involve only 2 layers (the mucosa and submucosa) of the bowel wall. Vascular access through the circular muscular layer of the bowel wall creates weak points that may result in diverticular formation. Conversely, true diverticula are the result of protrusion through all 3 layers (mucosa, submucosa, and muscularis externa) of the bowel wall and are commonly congenital [14]. The case we present is one of a rare true diverticulum with a concomitant GIST resulting in perforation. GISTs arise from the progenitors of smooth muscle cells, which are only present in true diverticula [1].

Initial presenting symptoms result from GIST protrusion into the GI lumen or abdominal cavity. Often the vascularity of the tumor results in bleeding. Subsequent signs and symptoms 
include anemia, tachycardia, fainting, abdominal pain, melena, hematemesis, and intestinal obstruction. The average tumor size is about $6 \mathrm{~cm}$ but may be much larger [2]. Imaging standards include first-line contrast-enhanced CT with second-line MRI only being preferred in patients where contrast is contraindicated or tumor location requires additional information. Endoscopy may be helpful for gastric tumors [2].

While GIST morphology is variable, common mutations exist that provide an easy basis for IHC diagnosis. About 95\% of GISTs stain for an activating mutation in KIT (encoding the c-KIT oncogene), and about $10-20 \%$ of GISTs are positive for PDGFRA (encoding for platelet-derived growth factor receptor tyrosine kinase) $[7,15]$. There are other known mutations, but KIT and PDGFRA are considered the main oncogenic mutations of interest in GISTs [16]. Tumor biopsy and staining is critical for optimal treatment, as GISTs presenting without KIT or PDGFRA mutations will respond poorly to tyrosine kinase inhibitors such as imatinib. Additionally, other mutations may indicate alternative treatment [2]. For further confirmatory diagnosis, IHC staining against DOG-1, which encodes a chloride channel protein called anoctamin 1, can be performed [17].

Surgery is the main treatment for most other small tumors, as is the case for the patient presented in this report. The cure rate is about $60 \%$ of patients with localized GIST [13]. The need for further treatment with the targeted drug imatinib (Gleevec ${ }^{\circledR}$ ), either before surgery (neoadjuvant) or after surgery (adjuvant therapy), depends on the risk of the GIST recurrence.

When given before surgery, imatinib, the most popular tyrosine kinase inhibitor, blocks c-KIT and PDGFRA and reduces tumor size or burden, therefore increasing the resectability of the GIST [18]. Treatment duration and response must be carefully monitored for optimal results. As for adjuvant therapy, imatinib is usually recommended for at least 3 years and has repeatedly shown improved recurrence-free survival compared to controls [19].

Spontaneous perforation of the GI diverticulum is rare. According to our literature review, this is the first case presentation of a GIST perforating through a diverticular structure within the terminal ileum, producing the unique symptoms observed in the patient. GISTs in other diverticular structures, such as Meckel's diverticulum, have been reported several times $[8,9]$. The mechanisms surrounding perforation of the GI wall are not fully understood. A popular theory involves neoplastic cells replacing the muscularis propria of the gut wall, resulting in a fragile diverticular structure [20]. Other possible explanations for perforation include necrosis of tumor cells and increased luminal pressure due to intestinal obstruction by the tumor [9].

While GISTs are an uncommon cancer of the GI tract, their presentation and management could be challenging. The coexistence of a GIST in a small bowel diverticulum is very rare, and in such a case, an oncologic segmental small intestine resection should be considered for successful treatment. Accurate risk stratification of GISTs is important to determine the need for adjuvant systemic treatments after surgery.

\section{Acknowledgment}

This article does not include any additional contributors to acknowledge.

\section{Statement of Ethics}

This study protocol was reviewed and deemed nonresearch by the WMU Homer Stryker M.D. School of Medicine Institutional Review Board. The study was conducted according to the principles of the World Medical Association Declaration of Helsinki. Written informed consent was obtained from the patient for publication of this case report and any accompanying images.

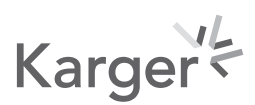




\section{Conflict of Interest Statement}

The authors have no conflicts of interest to declare.

\section{Funding Sources}

No funding was received for this study.

\section{Author Contributions}

Mackenna Senti, BS, is the primary author and researcher of this manuscript. Jairo Espinosa, MD, and Tania Torres, MD, are surgery residents who assisted in writing of the manuscript. Saad Shebrain, MD, MMM, was the principal investigator of this research and was involved in all steps of the process from case data collection to editing of the manuscript. All authors have read and approved the final manuscript.

\section{Data Availability Statement}

All data generated or analyzed during this study are included in this article. Further enquiries can be directed to the corresponding author.

\section{References}

1 Nilsson B, Bümming P, Meis-Kindblom JM, Odén A, Dortok A, Gustavsson B, et al. Gastrointestinal stromal tumors: the incidence, prevalence, clinical course, and prognostication in the preimatinib mesylate era: a population-based study in western Sweden. Cancer. 2005;103:821-9.

2 von Mehren M, Joensuu H. Gastrointestinal stromal tumors. J Clin Oncol. 2018;36:136-43.

3 Agaimy A, Wünsch PH, Hofstaedter F, Blaszyk H, Rümmele P, Gaumann A, et al. Minute Gastric Sclerosing Stromal Tumors (GIST Tumorlets) are common in adults and frequently show c-KIT mutations. Am J Surg Pathol. 2007; 31(1):113-20.

4 de Marco G, Roviello F, Marrelli D, de Stefano A, Neri A, Rossi S, et al. A clinical case of duodenal gastrointestinal stromal tumor with a peculiarity in the surgical approach. Tumori. 2005;91(3):261-3.

5 Rubin BP, Heinrich MC, Corless CL. Gastrointestinal stromal tumour. Lancet. 2007;369(9574):1731-41.

6 Emile JF, Brahimi S, Coindre JM, Bringuier PP, Monges G, Samb P, et al. Frequencies of KIT and PDGFRA mutations in the MolecGIST prospective population-based study differ from those of advanced GISTs. Med Oncol. 2012; 29(3):1765-72.

7 Ricci R. Syndromic gastrointestinal stromal tumors. Hered Cancer Clin Pract. 2016;14:15.

8 Ashoor AA, Barefah G. Unusual presentation of a large GIST in an extraintestinal site: a challenging diagnosis dilemma. BMJ Case Rep. 2020;13(2):229839.

9 Miyata S, Bliss DW. A gastrointestinal stromal tumor found in perforated Meckel's diverticulum. Surg Case Rep. 2016;2:67.

10 Nishida T, Hølmebakk T, Raut CP, Rutkowski P. Defining tumor rupture in gastrointestinal stromal tumor. Ann Surg Oncol. 2019;26(6):1669.

11 Demetri GD, Benjamin R, Blanke CD, Choi H, Corless C, DeMatteo RP, et al. NCCN Task Force report: optimal management of patients with gastrointestinal stromal tumor (GIST): expansion and update of NCCN clinical practice guidelines. J Natl Compr Canc Netw. 2004;2(Suppl 1):S1-26.

12 Ducimetière F, Lurkin A, Ranchère-Vince D, Decouvelaere AV, Péoc'h M, Istier L, et al. Incidence of sarcoma histotypes and molecular subtypes in a prospective epidemiological study with central pathology review and molecular testing. PLoS One. 2011;6(8):e20294.

13 Joensuu H, Vehtari A, Riihimäki J, Nishida T, Steigen SE, Brabec P, et al. Risk of recurrence of gastrointestinal stromal tumour after surgery: an analysis of pooled population-based cohorts. Lancet Oncol. 2012;13:265-74.

14 Maykel JA, Opelka FG. Colonic diverticulosis and diverticular hemorrhage. Clin Colon Rectal Surg. 2004;17(3): 195-204. 
15 Medeiros F, Corless CL, Duensing A, Hornick JL, Oliveira AM, Heinrich MC, et al. KIT-negative gastrointestinal stromal tumors: proof of concept and therapeutic implications. Am J Surg Pathol. 2004;28(7):889-94.

16 Corless CL, Barnett CM, Heinrich MC. Gastrointestinal stromal tumours: origin and molecular oncology. Nat Rev Cancer. 2011;11(12):865-78.

17 Lopes LF, West RB, Bacchi LM, van de Rijn M, Bacchi CE. DOG1 for the diagnosis of gastrointestinal stromal tumor (GIST): comparison between 2 different antibodies. Appl Immunohistochem Mol Morphol. 2010;18(4): 333-7.

18 Wang D, Zhang Q, Blanke CD, Demetri GD, Heinrich MC, Watson JC, et al. Phase II trial of neoadjuvant/adjuvant imatinib mesylate for advanced primary and metastatic/recurrent operable gastrointestinal stromal tumors: long-term follow-up results of radiation therapy oncology group 0132. Ann Surg Oncol. 2012;19:1074-80.

19 Joensuu H, Eriksson M, Sundby Hall K, Reichardt A, Hartmann JT, Pink D, et al. Adjuvant imatinib for high-risk GI stromal tumor: analysis of a randomized trial. J Clin Oncol. 2016;34(3):244-50.

20 Ikemura M, Kunita A, Miwa Y, Jimbo K, Mori K, Seto Y, et al. Gut wall replacing type of gastrointestinal stromal tumor presenting as a perforation of the ileal diverticulum. Pathol Res Pract. 2015;211(11):892-5. 